\title{
A review on the micro energy harvester in Structural Health Monitoring (SHM) of biocomposite material for Vertical Axis Wind Turbine (VAWT) system: a Malaysia perspective
}

\begin{abstract}
The usage of wind energy as a form of renewable energy is becoming increasingly popular year by year. This technology has been applied widely in several regions in the world and already reached maturity in terms of technology, infrastructure and cost competitiveness. The performance of the wind turbine system depends upon factors such as design, aerodynamic performance and material selection. Thus, Structural Health Monitoring (SHM) has become crucial in evaluating the performance of wind turbine in real time. Furthermore, the application of smart material in SHM can be utilized as micro energy harvester as well. Nonetheless, the application of SHM in Malaysia's climate for wind turbine is still premature, especially in the approach biocomposites material towards its blade system. Several issues are highlighted in this paper such as Vertical Axis Wind Turbine (VAWT), biocomposites material selection and the issue in the micro energy harvester as well. The issues are discussed and compared with the recent finding in this review. Several recommendations are suggested for future studies in benefiting the Malaysian especially on the application of wind energy to promote better green technology for tomorrow.
\end{abstract}

Keyword: Biocomposite fiber; Vertical Axis Wind Turbine; Piezoelectric; Micro energy harvester 